\title{
Evaluation of the efficacy of neoadjuvant chemotherapy for breast cancer using diffusion-weighted imaging and dynamic contrast-enhanced magnetic resonance imaging
}

\author{
H. D. XU*, Y. Q. ZHANG \\ Department of Radiology, Ningbo Medical Center Lihuili Eastern Hospital, Ningbo City 315040, P.R. China \\ ${ }^{\star}$ Correspondence: xuhaidong50@126.com
}

Received August 18, 2016/ Accepted November 6, 2016

\begin{abstract}
This study aims to investigate the predictive values of diffusion-weighted imaging (DWI) and dynamic contrast-enhanced magnetic resonance imaging (DCE-MRI) in evaluating the efficacy of neoadjuvant chemotherapy (NAC) for breast cancer. Between September 2011 and December 2014, a total of 174 patients with locally advanced breast cancer treated with NAC were selected for this study. Conventional CT and MRI examinations (DWI and DCE-MRI) were performed in all patients before NAC (T0), after the first course (T1) and after the whole course (T2) of NAC. According to the response evaluation criteria in solid tumors (RECIST), patients were divided into the effective [complete response (CR) + partial response (PR)] and ineffective groups [stable disease (SD) + disease progression (DP)]. The Apparent diffusion coefficient (ADC), maximum tumor diameter, the early-phase enhancement rate $(\mathrm{Ee})$, maximal enhanced rate of tumor $\left(\mathrm{E}_{\max }\right)$, maximal linear slope $\left(\mathrm{S}_{\max }\right)$, maximal excretion rate $\left(\mathrm{E}_{\text {wash }}\right)$, signal intensity $(\mathrm{SI})$, maximal signal rise velocity $\left(\mathrm{V}_{\max }\right)$ and area under the curve (AUC) of Cho before and after NAC were calculated. Receiver operating characteristic (ROC) curve was drawn and the AUC of change rate of $\mathrm{ADC}$ values and semi-quantitative parameter were utilized to analyze the diagnostic performance of them for evaluating the efficacy of NAC for breast cancer. There were 135 patients in the effective group, with 52 cases of CR and 83 cases of PR; 39 patients were in the ineffective group, with 14 cases of PD and 25 cases of SD. The effective rate of NAC for breast cancer was $77.6 \%$. The ADC values of the two groups significantly increased but the maximum tumor diameter, E e, $\mathrm{E}_{\text {max }}, \mathrm{S}_{\text {max }}, \mathrm{E}_{\text {wash }}$ and AUC of Cho greatly decreased. The effective group had higher ADC values, its change rate and tumor regression rate than the ineffective group. However, the maximum tumor diameter, E e, $\mathrm{E}_{\text {max }}, \mathrm{S}_{\text {max }}, \mathrm{E}_{\text {wash }}$, AUC of Cho, SI and $\mathrm{V}_{\max }$ in the effective group were remarkably lower than those in the ineffective group. The change rate of ADC ${ }_{\text {mean }}$ achieved the highest evaluation efficiency with AUC of 0.920 , sensitivity of $80.0 \%$ and specificity of $94.9 \%$. The optimal critical value was $36.49(\times 10-3 \mathrm{~mm} 2 / \mathrm{s})$. In conclusion, these results demonstrated that the change rate of ADC ${ }_{\text {mean }}$ values and $\mathrm{E}$ e could be promising tools for evaluating the efficacy of NAC in patients with breast cancer.
\end{abstract}

Key words: diffusion-weighted imaging, dynamic contrast-enhanced, magnetic resonance imaging, breast cancer, neoadjuvant chemotherapy, efficacy, evaluation

Breast cancer is the most common malignant tumor and one of the leading causes of death in women in the world [1]. According to literature, the incidence rates of breast cancer are higher in developed countries than those in developing countries [2]. It is estimated that about 3.2 million people will be diagnosed with breast cancer each year by 2050 worldwide [3]. Breast cancer is a complex and multifactorial disease, which is affected by combined effects of environmental and inherited factors [4]. It is characterized by starting with hyperplasia, in distinct steps, in situ ductal and invasive carcinoma then evolv- ing into a deadly metastatic disease [5]. Recently, it has been shown that neoadjuvant chemotherapy (NAC) can reduce both disease recurrence and mortality, and improve survival especially identical long-term survival in patients with breast cancer [6]. Despite its potential advantages, there are barriers that limit the broad adoption of NAC in practice, including the higher complexities of monitoring tumor response and cancer progression during therapy [7]. Magnetic resonance imaging (MRI) is now used to predict pathological complete response (pCR) and clinical outcomes in patients treated with NAC [8]. 
MRI is an established supplementary technique for diagnosis and evaluation of diseases in organ system, and it is a promising method in detection, localization and staging of cancers, including breast cancer [9]. DWI and DCE-MRI are two commonly used methods in MRI. DWI is a functional imaging which can detect the motion of water molecules in living things and water diffusion which is assessed by calculation of ADC [10]. DCE-MRI has the potential to provide information about the microenvironment, angiogenesis and the biologic aggressiveness of the tumor [11]. Document revealed that DWI and DCE-MRI had been proved to be superior to clinical caliper measurement, supplemented with ultrasound and mammography, but it was controversial, and it is still remained to be established whether and how MRI parameters such as tumor size, optimal time points, cell membrane integrity, water diffusion were associated with PCR and clinical outcomes in NAC $[8,12]$. Thus, the purpose of this study is to investigate the predictive values of DWI and DCE-MRI in evaluating the efficacy of NAC for breast cancer by comparing the changes in tumor size, ADC, tumor regression rate and semi-quantitative parameters in the two functional techniques at different time points.

\section{Materials and methods}

Study subjects. A total of 174 patients with locally advanced breast cancer [stage II/III according to International Union against Cancer (UICC) tumor node metastasis (TNM) Classification] [13] treated with NAC firstly and surgical resection in Ningbo Medical Center Lihuili Eastern Hospital were recruited from September 2011 to December 2014. All the patients were females (28 to 64 years old) with an average age of $45.7 \pm 7.8$ years, diagnosed with breast cancer by ultrasound and core needle biopsy $(\mathrm{CNB})$ before chemotherapy (category: breast imaging reporting and data system (BI-RADS) 5) [14]. There were a number of 154 patients with infiltrating ductal carcinoma (IDC), 3 with infiltrating ductal and intraductal carcinoma, 5 with infiltrating ductal and mucinous adenocarcinoma and 12 with infiltrating lobular carcinoma (ILC). All of the tumor diameters were no less than $2.0 \mathrm{~cm}$. All the patients were treated with for 4 courses (21 days for each course) and CET (cyclophosphamide plus epirubicin and taxotere) were included in the chemotherapy regimens. Three of four weeks after the chemotherapy regimens, the surgical resection was performed. Patients were excluded for the following reasons: a. who gave up treatment or changed the regimens; $b$. who were confirmed as patients without breast cancer in final pathology; c. who had disorders in heart, lung, and liver or endocrine diseases or history of malignant tumors; $d$. who undergone radio-chemotherapy; e. who had contraindications to MRI. The clinical data were obtained based on approval from the Ethics Committee of Ningbo Medical Center Lihuili Eastern Hospital and informed consents were obtained from all the study subjects.

Scanning methods. Signa Excite HD 3.0 T MR scanner (GE Medical Systems, Milwaukee, WI, USA) with an 8-channel phased-array coil for breast was adopted. Each examination included conventional CT, DWI and DCE-MRI. Patients were in prone position to make their bilateral breast prolapse and hang in the coil naturally. Conventional CT: Conventional axial, sagittal and coronal position were scanned, and conventional sequences included fast spin echo (FSE) T1-weighted images (T1WI) [repetition time (TR) $8.6 \mathrm{~ms}$; echo time (TE) $4.7 \mathrm{~ms}$; slice thickness $1 \mathrm{~mm}$; slice gap $0.2 \mathrm{~mm}$; field of view (FOV) $320 \mathrm{~mm} \times 320 \mathrm{~mm}$; number of excitations 1] and fat-suppression T2-weighted images sequence (TR $5600 \mathrm{~ms}$; TE $56 \mathrm{~s}$; slice thickness $4 \mathrm{~mm}$; slice gap $1 \mathrm{~mm}$; FOV $340 \mathrm{~mm}$ $\times 340 \mathrm{~mm}$; number of excitations 2); slice thickness of fatsuppression FSE T2WI on sagittal position was $4 \mathrm{~mm}$ which was seamless. Axial DWI was performed using single-shot echo-planar imaging (SS-EPI) (TR $4000 \mathrm{~ms}$; TE $81 \mathrm{~ms}$; b value $0 \mathrm{~s} / \mathrm{mm}^{2}$ and $800 \mathrm{~s} / \mathrm{mm} 2$; slice thickness $4 \mathrm{~mm}$; seamless scanning; number of excitations 4). DCE-MRI sequence of bilateral breast was performed using an isotropic high resolution FLASH 3D T1 image volume and the parameters were the same with T1WI. A total of 10 times for $600 \mathrm{~s}$ sagittal scanning with mask was carried out (1 phase of post mask plus 9 phase of contrast enhancement). Contrast medium was $0.2 \mathrm{mmol} /$ $\mathrm{kg}$ gadolinium diethylene triaminepenta acetate (Gd-DTPA) which was injected into ulnar vein with current velocity of $2.5 \mathrm{ml} / \mathrm{s}$. After contrast agent bolus, $20 \mathrm{ml}$ normal saline was infused through the vein.

Image analysis. The obtained MRI data were analyzed using double-blind method by 2 experienced radiologists working on GE ADW4.4 workstation with Functool function package. Maximal diameter of breast cancer mass was used for comparing different techniques and $b$ value was entered to obtain the required experimental ADC, DWI and timeintensity curve (TIC).

Data measurement. Referring to MRI T2WI and fatsuppression T2WI, ADC map was firstly reconstructed on the workstation software based on DWI map $\left(b=0 \mathrm{~s} / \mathrm{mm}^{2}\right.$ and $b=800 \mathrm{~s} / \mathrm{mm}^{2}$ ). ADC value was measured under the condition where the location of the tumor on DWI map was corresponded to ADC map. Three largest breast cancer masses were selected, 4 regions (liquefactive necrosis and marginal tissues were excluded) with homogeneous signal intensity were measured and the area of each region of interest (ROI) was $10 \sim 15 \mathrm{~mm}^{2}$ and $20 \sim 30 \mathrm{dpi}$. The liquefactive necrosis and tumor marginal tissue were excluded. The number of measured ROI values was at least $12 . \mathrm{ADC}_{\text {mean }}$ of each lesion was obtained from the average $\mathrm{ROI}$ value and the $\mathrm{ADC}_{\text {min }}$ of each lesion was calculated based on averaging the minimal ADC values of the three masses. The time-points of measurement included $\mathrm{T}_{0}$ (before NCA), $\mathrm{T}_{1}$ (2-5 days after the first course of NAC) and $\mathrm{T}_{2}$ (3-4 weeks after whole course of NAC). The relevant ADC mean $, \mathrm{ADC}_{\text {mean1 }}, \mathrm{ADC}_{\text {mean2 } 2}, \mathrm{ADC}_{\text {min }}, \mathrm{ADC}_{\text {min1 }}$ and $\mathrm{ADC}$ min2 $_{\text {were }}$ wer calculated. The change rate $\left(\triangle \mathrm{ADC} \mathrm{mean}_{\text {min }} \%\right)$ of $\mathrm{ADC}{ }_{\text {mean } / \mathrm{min}}$ at $\mathrm{T}_{1}$ and $\mathrm{T}_{2}$ over $\mathrm{T}_{0}$ was obtained. $\triangle \mathrm{ADC} \%=\left(\mathrm{ADC}_{\mathrm{Tn}}-\mathrm{ADC}_{\mathrm{T}} \mathrm{m}\right) /$ $\mathrm{ADC}_{\mathrm{TO}} \times 100 \%, \mathrm{ADC}_{\mathrm{T} 0}$ referred to the $\mathrm{ADC}$ value before NAC and $A D C{ }_{T n}$ referred to the $A D C$ value at relevant $T_{n}$. 
Masses observed on the first phase enhanced image were divided into regular, lobulated and irregular shape. Maximal axial diameter of tumor was measured on axial position of maximal diameter of the largest lesion for 3 times and the mean value was calculated to obtain the maximum tumor diameter $(\mathrm{d})$. The tumor regression rate $\Delta \mathrm{d}_{\mathrm{n}} \%=\left(\mathrm{d}_{\mathrm{T} 0}-\mathrm{d}_{\mathrm{Tn}}\right) / \mathrm{d}_{\mathrm{T} 0}$ $\times 100 \%, \mathrm{~d}_{\mathrm{T} 0}$ referred to $\mathrm{d}$ value before NAC and $\mathrm{d}_{\mathrm{Tn}}$ referred to the $d$ value at relevant $\mathrm{T}_{\mathrm{n}}$.

According to the TIC map, semi-quantitative parameters were calculated. Early enhanced rate of tumor $(\mathrm{Ee})=[$ signal intensity $(\mathrm{SI})_{\text {second }}-\mathrm{SI}_{\text {pre }}$ ]/SI $\times 100 \%$; maximal enhanced rate of tumor $\left(\mathrm{E}_{\max }\right)=\left(\mathrm{SI}_{\text {max }}^{\text {pre }}-\mathrm{SI}_{\mathrm{pre}}^{\mathrm{pre}}\right) / \mathrm{SI} \mathrm{pre}_{\mathrm{pr}} \times 100 \%$; maximal linear slope $\left(\mathrm{S}_{\max }\right)=\left(\mathrm{SI}_{\text {end }}-\mathrm{SI}_{\text {prior }}\right) /\left[\mathrm{SI} \underset{\text { pre }}{ } \times\left(\mathrm{T}_{\text {end }}-\mathrm{T}_{\text {prior }}\right)\right] \times 100 \%$; maximal excretion rate $\left(\mathrm{E}_{\text {wash }}^{\text {prior }}\right)=\left(\mathrm{SI}_{\text {max }}^{\text {pre }}-\mathrm{SI}_{\text {last }}\right) / \mathrm{SI}_{\text {max }}^{\text {prior }} \times 100 \%$. Rise rate of lesion signal intensity SI $=\left[\left(\mathrm{SI}_{\text {post }}-\mathrm{SI}_{\text {pre }}\right) / \mathrm{SI}_{\text {pre }}\right]$ $\times 100 \%$. Maximal signal rise velocity $\mathrm{V}_{\max }=\left[\left(\mathrm{SI}_{\max }-\mathrm{SI}_{\mathrm{pre}}\right) / \mathrm{T}\right.$

$\left.-\mathrm{T}_{\text {pre }}\right] \times 100 \%$. AUC of choline-containing compounds (Cho) was obtained from single- and multi- voxel spectroscopy. SI pre represented signal value before enhancement, SI second meant signal intensity value of the second sequence after enhancement, SI ${ }_{\text {max }}$ referred to maximal signal value on TIC after enhancement, SI last represented signal intensity of the last sequence after enhancement, SI post meant signal intensity after chemotherapy, SI end and SI prior referred to signal intensity value of the two time points with maximal difference value at rapid increasing stage on $\mathrm{TIC}, \mathrm{T}$ end and $\mathrm{T}$ prior reflected the corresponding time points between SI ${ }_{\text {end }}$ and SI ${ }_{\text {prior }}$.

Efficacy evaluation. According to the maximum tumor diameter after NAC, patients were divided into four levels, including CR, PR, PD and SD according to RECIST [15]. CR: tumor completely disappeared after NAC; PR: maximum tumor diameter decreased by at least 30\%; PD: maximum tumor

Table 1. Clinical data of the breast cancer patients with different efficacy

\begin{tabular}{lccc}
\hline Variables & $\begin{array}{c}\text { Effective } \\
(\mathrm{n}=135)\end{array}$ & $\begin{array}{c}\text { Ineffective } \\
(\mathrm{n}=39)\end{array}$ & $P$ value \\
\hline Age (year) & $45.5 \pm 8.0$ & $46.5 \pm 7.0$ & 0.481 \\
Tumor diameter (cm) & $4.14 \pm 1.26$ & $3.78 \pm 0.95$ & 0.097 \\
TNM staging (n) & & & 0.674 \\
$\quad$ II & 71 & 22 & \\
III & 64 & 17 & \\
Histological classification (n) & & & 0.816 \\
IDC & 119 & 35 & \\
IDC plus IC & 3 & 0 & \\
IDC plus MA & 4 & 1 & \\
ILC & 9 & 3 & \\
Lymphatic metastasis before & & & \\
chemotherapy (n) & & & \\
Yes & 77 & 26 & \\
$\quad$ No & 58 & 13 & \\
Elevated TM CA153 (u/ml) & $41.98 \pm 10.12$ & $44.03 \pm 12.26$ & 0.290 \\
\hline
\end{tabular}

Note: TNM, tumor node metastasis; IDC, infitrating ductal carcinoma; IC, intraductal carcinoma; MA, mucinous adenocarcinoma; ILC, infiltrating lobular carcinoma; TM, tumor maker. diameter increased by at least 20\%; SD: tumor changed between PR and PD. All patients were assigned into the effective (CR plus $\mathrm{PR}$ ) group and ineffective (SD plus $\mathrm{PD}$ ) group. (CR + $\mathrm{PR}) /$ (total number of patients) represented total response rate.

Statistical analysis. Data was analyzed by SPSS 21.0 (SPSS Inc., Chicago, IL, USA). Count data were presented as percentage and measurement data were shown as ' $\mathrm{x} \pm \mathrm{s}$. Comparisons of patients' age, TNM staging, histological classification and lymphatic metastasis were conducted using chi-square test. ADC values, maximum tumor diameter, semi-quantitative parameters, and other parameters were analyzed and compared using $t$-test before and after NAC. The effective group was positive and the ineffective group was negative, based on which ROC curve was drawn to calculate AUC of the change rate of $\mathrm{ADC}$ value and the semi-quantitative parameters. Thus, diagnostic efficiency in evaluating of the efficacy of NAC for breast cancer was analyzed and the optimal critical value for diagnosis was obtained.

\section{Results}

Efficacy of NAC for the breast cancer patients. The patients $(\mathrm{n}=174)$ were divided into two groups according to RECIST: 135 cases of effective (CR: 52 and PR: 83); 39 cases of ineffective (PD: 14 and SD: 25). The effective rate of NAC was $77.6 \%$. There were no differences in age, tumor diameter, TNM staging, histological classification, pretreatment lymphatic metastasis or tumor maker (TM) between effective and ineffective groups (all $P>0.05$ ) (Table 1).

Comparison of ADC values and its rate of change of the breast cancer patients at different time points. The ADC value in the two groups showed a trend of gradual increase after NAC. The ADC mean and ADC min values significantly increased in the two groups after NAC (both $P<0.05$ ). The $\mathrm{ADC}_{\text {mean }}$ and $\mathrm{ADC}{ }_{\text {min }}$ values after the whole course of NAC were evidently higher than those after the first course of NAC (both $P<0.05$ ). There were no significant differences in the $\mathrm{ADC}_{\text {mean }}, \mathrm{ADC}_{\text {min }}$ values and rates of change of $\mathrm{ADC}_{\text {mean }}$ or $\mathrm{ADC}_{\text {min }}$ values between two groups before and after the first course of NAC $(P>0.05)$; the $\mathrm{ADC}_{\text {mean }}, \mathrm{ADC}_{\text {min }}$ values and rates of change of $\mathrm{ADC}_{\text {mean }}$ and $\mathrm{ADC}_{\text {min }}$ values in the effective group were greatly higher than those in the ineffective group after whole course of NAC (all $P<0.05$ ) (Table 2).

Comparison of maximum tumor diameter and tumor regression rate of the breast cancer patients in the effective and ineffective groups at different time points. The maximum tumor diameter in the two groups showed a trend of gradual decrease after NAC. In the two groups, the maximum tumor diameter significantly decreased at 3 time points before and after NAC (all $P<0.05$ ); the tumor regression rate greatly increased after the whole course of NAC in comparison to that after the first course of NAC (both $P<$ $0.05)$. There was no significant difference in the maximum tumor diameter and tumor regression rate between the two groups before and after the first course of NAC $(P>0.05)$. 
Compared with the ineffective group, the effective group showed significantly smaller maximum tumor diameter and higher tumor regression rate after the whole course of NAC (both $P<0.05$ ) (Table 3).

Table 2. The ADC values and the rates of changes of ADC values (T0, T1, T2) of the breast cancer patients before and after neoadjuvant chemotherapy

\begin{tabular}{|c|c|c|c|}
\hline ADC values & $\begin{array}{l}\text { Effective group } \\
\quad(\mathrm{n}=135)\end{array}$ & $\begin{array}{l}\text { Ineffective group } \\
\qquad(\mathrm{n}=39)\end{array}$ & $P$ value \\
\hline $\mathrm{ADC}_{\text {mean }}$ & $0.84 \pm 0.15$ & $0.86 \pm 0.09$ & 0.268 \\
\hline $\mathrm{ADC}_{\text {mean } 1}$ & $0.95 \pm 0.19^{*}$ & $0.97 \pm 0.11^{*}$ & 0.453 \\
\hline $\mathrm{ADC}_{\text {mean } 2}$ & $1.27 \pm 0.31^{\star \#}$ & $1.08 \pm 0.15^{\star \#}$ & $<0.001$ \\
\hline$\Delta \mathrm{ADC}_{\text {meanl }} \%$ & $13.13 \pm 3.08$ & $12.81 \pm 1.64$ & 0.387 \\
\hline$\Delta \mathrm{ADC}_{\text {mean } 2} \%$ & $49.09 \pm 12.34^{\mathrm{a}}$ & $25.65 \pm 7.50^{\mathrm{a}}$ & $<0.001$ \\
\hline $\mathrm{ADC}_{\text {min }}$ & $0.75 \pm 0.16$ & $0.78 \pm 0.07$ & 0.092 \\
\hline $\mathrm{ADC}_{\min 1}$ & $0.81 \pm 0.19^{*}$ & $0.84 \pm 0.08^{*}$ & 0.154 \\
\hline $\mathrm{ADC}_{\min 2}$ & $1.11 \pm 0.30^{* \#}$ & $0.98 \pm 0.13^{\text {*\# }}$ & $<0.001$ \\
\hline$\Delta \mathrm{ADC}_{\min 1} \%$ & $8.33 \pm 2.07$ & $8.07 \pm 0.84$ & 0.243 \\
\hline$\Delta \mathrm{ADC}_{\min 2} \%$ & $46.94 \pm 13.39^{\#}$ & $25.26 \pm 6.32^{*}$ & $<0.001$ \\
\hline
\end{tabular}

Note: ADC ${ }_{\text {mean }}$, average ADC before NAC; ADC ${ }_{\text {mean } 1}$, average ADC after first course of NAC; ADC ${ }_{\text {mean } 2}$, average ADC after complete course of NAC; $\triangle \mathrm{ADC} \quad \%$, change rate of average ADC after first course of NAC; $\triangle \mathrm{ADC}$ mean $2 \%$, the rate of change of average ADC after complete course of NAC; ADC ${ }_{\text {min }}$, minimal ADC before NAC; A DC ${ }_{\text {minl }}$, minimal ADC after first course of NAC; $\mathrm{ADC}_{\text {min }_{2}}$, minimal ADC after complete course of NAC; $\triangle \mathrm{ADC}_{\min 1} \%$, change rate of minimal ADC after first course of NAC; $\triangle \mathrm{ADC}{ }_{\min 2} \%$, change rate of minimal $\mathrm{ADC}$ after complete course of NAC; ${ }^{*}$, compared with the ADC value before NAC, $P<0.05$; ${ }^{*}$, compared with the ADC value after the first course of NAC, $P<0.05$.
Comparison of semi-quantitative parameters of the breast cancer patients in the effective and ineffective groups at different time points. The $\mathrm{Ee}, \mathrm{E}_{\text {max }}, \mathrm{S}_{\text {max }}, \mathrm{E}_{\text {wash }}$ and AUC of Cho of the effective and ineffective groups significantly decreased (all $P<0.05$ ). The effective group had greatly lower SI and $\mathrm{V}_{\text {max }}$ after whole course of NAC in comparison to those after the first course of NAC (all $P<0.05$ ). There was no significant difference in Ee, $E_{\text {max }}, S_{\text {max }}, E_{\text {wash }}, A U C$ of Cho, SI and $\mathrm{V}_{\text {max }}$ between the two groups before and after the first course of NAC (all $P>0.05$ ). Compared with the ineffective group, the effective group had remarkably lower Ee, $\mathrm{E}_{\text {max }}, \mathrm{S}_{\text {max }}$, $\mathrm{E}_{\text {wash }}$, AUC of Cho, SI and $\mathrm{V}_{\text {max }}$ values after the whole course of NAC (all $P<0.05)$ (Table 4).

Table 3. Maximum tumor diameter and its change rate at 3 time points before and after neoadjuvant chemotherapy (NAC) $(\mathrm{cm})$

\begin{tabular}{lccc}
\hline Maximum tumor diameter & $\begin{array}{c}\text { Effective group } \\
(\mathrm{n}=135)\end{array}$ & $\begin{array}{c}\text { Ineffective } \\
\text { group }(\mathrm{n}=39)\end{array}$ & $P$ value \\
\hline Before NAC & $4.14 \pm 1.26$ & $3.78 \pm 0.95$ & 0.097 \\
After first course of NAC & $3.82 \pm 1.15^{*}$ & $3.50 \pm 0.83^{*}$ & 0.057 \\
After whole course of NAC & $1.61 \pm 1.54^{* *}$ & $2.88 \pm 0.77^{* *}$ & $<0.001$ \\
$\Delta \mathrm{d}_{1} \%$ & $7.85 \pm 1.96$ & $7.03 \pm 2.49$ & 0.062 \\
$\Delta \mathrm{d}_{2} \%$ & $68.56 \pm 27.98^{*}$ & $23.81 \pm 4.39^{*}$ & $<0.001$ \\
\hline
\end{tabular}

Note: $\Delta \mathrm{d}_{1} \%$, rate of change of maximum diameter at early stage; $\Delta \mathrm{d}_{2} \%$, rate of change of maximum axial diameter after whole chemotherapy; ${ }^{*}$, compared with before NAC, $P<0.05$; \#, compared with after the first course of NAC, $P<0.05$.

Table 4. The changes of semi-quantitative parameters in tumor $(\mathrm{T} 0, \mathrm{~T} 1, \mathrm{~T} 2)$ of the breast cancer patients before and after neoadjuvant chemotherapy

\begin{tabular}{|c|c|c|c|c|}
\hline Parameter & Time point & Effective group $(\mathrm{n}=135)$ & Ineffective group $(\mathrm{n}=39)$ & $P$ value \\
\hline \multirow{3}{*}{$\mathrm{Ee}$} & Before NAC & $165.19 \pm 38.60$ & $159.42 \pm 44.31$ & 0.428 \\
\hline & After first course of NAC & $147.25 \pm 39.08^{*}$ & $155.64 \pm 41.27^{*}$ & 0.245 \\
\hline & After whole course of NAC & $92.83 \pm 32.81^{* \#}$ & $151.93 \pm 42.09^{* \#}$ & $<0.001$ \\
\hline \multirow{3}{*}{$\mathrm{E}_{\text {max }}$} & Before NAC & $196.53 \pm 42.15$ & $187.94 \pm 39.14$ & 0.256 \\
\hline & After first course of NAC & $184.57 \pm 43.02^{*}$ & $175.61 \pm 40.46^{*}$ & 0.247 \\
\hline & After whole course of NAC & $145.12 \pm 31.57^{* \#}$ & $170.41 \pm 37.30^{* \#}$ & $<0.001$ \\
\hline \multirow{3}{*}{$\mathrm{S}_{\text {max }}$} & Before NAC & $94.63 \pm 32.43$ & $89.89 \pm 40.08$ & 0.448 \\
\hline & After first course of NAC & $75.77 \pm 37.37^{*}$ & $79.56 \pm 36.37^{*}$ & 0.576 \\
\hline & After whole course of NAC & $28.59 \pm 15.28^{* \#}$ & $69.41 \pm 33.57^{* *}$ & $<0.001$ \\
\hline \multirow{3}{*}{$\mathrm{E}_{\text {wash }}$} & Before NAC & $7.56 \pm 3.58$ & $6.83 \pm 3.64$ & 0.261 \\
\hline & After first course of NAC & $5.44 \pm 3.54^{*}$ & $6.25 \pm 3.20^{*}$ & 0.200 \\
\hline & After whole course of NAC & $1.41 \pm 0.96^{* *}$ & $4.12 \pm 2.41^{* \text { \# }}$ & $<0.001$ \\
\hline \multirow{3}{*}{ AUC of Cho } & Before NAC & $18.25 \pm 10.46$ & $20.70 \pm 15.26$ & 0.351 \\
\hline & After first course of NAC & $10.87 \pm 7.15^{*}$ & $13.71 \pm 10.27^{*}$ & 0.112 \\
\hline & After whole course of NAC & $3.31 \pm 2.85^{* *}$ & $11.27 \pm 9.81^{* \#}$ & $<0.001$ \\
\hline \multirow{2}{*}{ SI } & After first course of NAC & $119.16 \pm 38.11$ & $114.44 \pm 28.50$ & 0.474 \\
\hline & After whole course of NAC & $90.92 \pm 23.38^{\#}$ & $114.91 \pm 26.09^{\#}$ & $<0.001$ \\
\hline \multirow{2}{*}{$\mathrm{V}_{\max }$} & After first course of NAC & $210.43 \pm 76.60$ & $236.25 \pm 67.57$ & 0.059 \\
\hline & After whole course of NAC & $149.35 \pm 71.34^{\#}$ & $236.67 \pm 68.61^{\#}$ & $<0.001$ \\
\hline
\end{tabular}

Note: Ee, early enhanced rate of tumor; $\mathrm{E}_{\text {max }}$, maximal enhanced rate of tumor; $\mathrm{S}_{\text {max }}$, maximal linear slope; $\mathrm{E}_{\text {wash }}$, maximal excretion rate; $\mathrm{AUC}$, area under the concentration-time curve; Cho, choline-containing compounds; $\mathrm{SI}$, signal intensity; $\mathrm{V}_{\text {max }}$, maximal signal rise velocity; ${ }^{*}$, compared with before NAC, $P<$ 0.05 ; $^{*}$ compared with after the first course of NAC, $P<0.05$. 
A

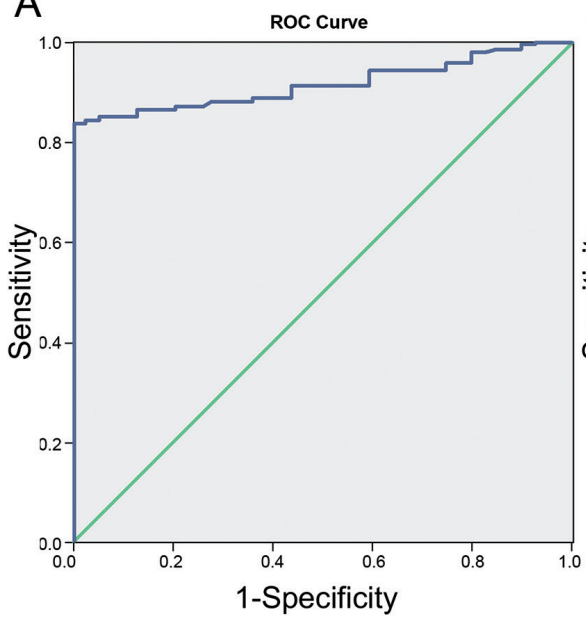

D

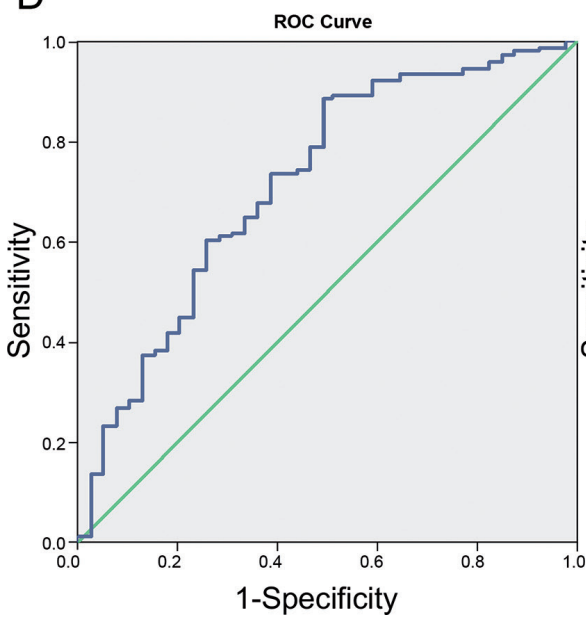

B

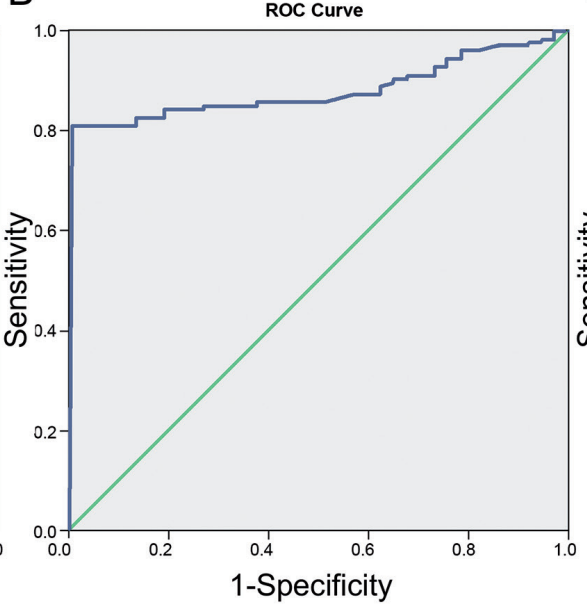

E

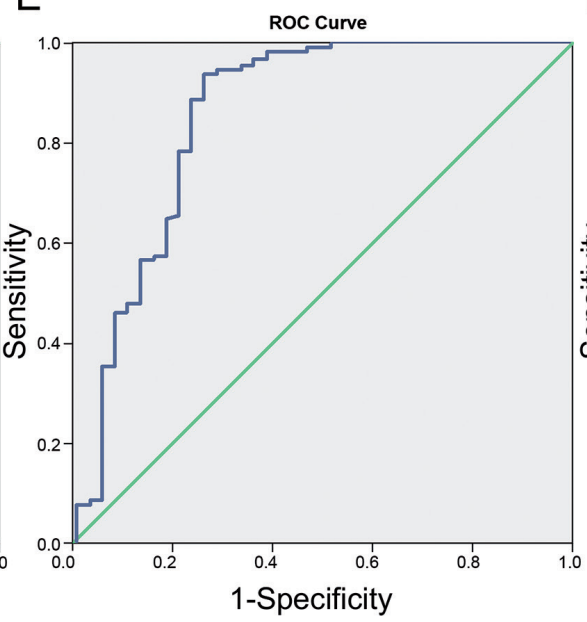

C

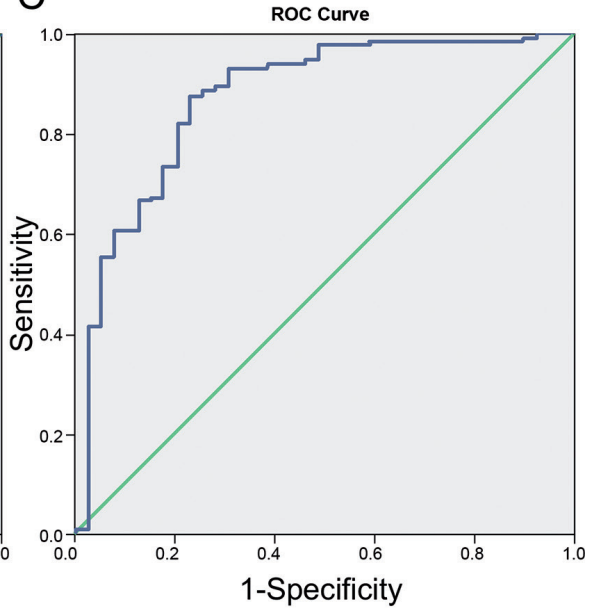

$\mathrm{F}$

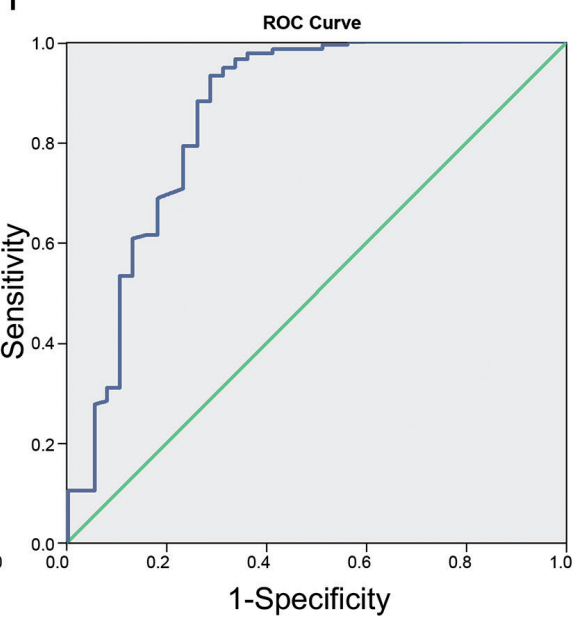

Figure 1. ROC curves of radiological parameters in evaluating the efficacy of neoadjuvant chemotherapy (AUC $>0.7)$.

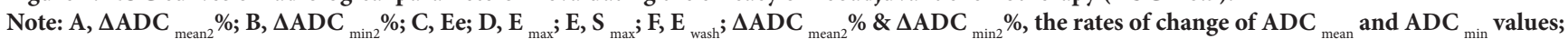
$\mathrm{Ee}$, the early-phase enhancement rate; $\mathrm{E}_{\max }$, maximum enhancement rate; $\mathrm{S}_{\max }$, maximum slope value; $\mathrm{E}_{\text {wash }}$, maximum excretion rate; $\mathrm{ROC}$, receiver operating characteristic.

Sensitivity and specificity of ADC values and semi-quantitative parameters in evaluating the efficacy of NAC for breast cancer. As seen in Table 5, rate of change of ADC value and semiquantitative parameters had no diagnostic efficiency before NAC and after first course of NAC $(P>0.05)$, but had higher diagnostic efficiency after whole course of NAC (AUC > 0.5). The change rate of $\mathrm{ADC}$ mean had the highest diagnostic efficiency: $\mathrm{AUC}=$ 0.920 , sensitivity: $80.0 \%$ and specificity: $94.9 \%$. Optimal critical value was $36.49(\times 10-3 \mathrm{~mm} 2 / \mathrm{s})$. The AUC of $\triangle \mathrm{ADC}_{\min 2} \%$ and Ee were 0.880 and 0.873 , respectively. In Figure 1, ROC curve showed the parameters with AUC value $>0.7$ after NAC.

\section{Discussion}

In this study, we explored the predictive value of DWI and DCE-MRI in evaluating for the efficacy of NAC for breast cancer patients. The patients were treated with different chemotherapy regimens and ADC value, tumor regression rate, and semi-quantitative parameters were analyzed. Our study showed that $\mathrm{ADC}$ mean, $\mathrm{ADC}_{\text {min }}$, and tumor regression rate increased significantly in the effective group than those in the ineffective group after whole course of NAC and the parameters of both DWI and DCE-MRI after four courses of NAC yielded a better performance (measured by ROC curve) than either before the NAC before or after the first course of NAC. Fangberget et al. reported that the ADC and ADC ${ }_{\text {min }}$ after four courses of NAC showed a significant difference between the patients in the effective group and ineffective group [12]. It was found that increase in ADC value was the consequence of cellular damage and necrosis. During the apoptotic process, membrane blebbing and cell lysis resulted in initial cellular swelling followed by reduction 
Table 5. The AUC and diagnostic performance of all parameters and the rate of changes of values of the breast cancer patients before and after neoadjuvant chemotherapy



Note: AUC, area under the concentration-time curve; $\triangle \mathrm{ADC}$ mean $\%$, change rate of average ADC after first course of NAC; $\triangle \mathrm{ADC}$ mean $\%$, change rate of average $\mathrm{ADC}$ after complete course of NAC; $\mathrm{ADC}_{\min 1} \%$, change rate of minimal ADC after first course of NAC; ADC ${ }_{\text {min } 2} \%$, change rate of minimal ADC after whole cycle of NAC; Ee, early enhanced rate of tumor; $\mathrm{E}_{\text {max }}$, maximal enhanced rate of tumor; $\mathrm{S}_{\text {max }}$, maximal linear slope; $\mathrm{E}_{\text {wash }}$, maximal excretion rate.

in cellular volume. Then, the movement of water molecules was enhanced, and water also moved more freely between the intra- and extracellular compartments [16]. One study related to rectal cancer reveled that response patients had lower ADC values at presentation than non-response patients; in addition, a strong negative correlation was found between pretreatment $\mathrm{ADC}_{\text {mean }}$ value and percentage size change of the tumor after chemotherapy [17]. The reason might be: high cellular proliferation causes an increase in cellular density which could restrict the diffusion of water molecules thus reducing ADC values and increasing tumor signal intensities on DWI [12]. This result indicated that before the NAC ADC mean value could be used as a biomarker to predict tumor regression rate.

Yuan et al. conducted a meta-analysis to evaluate the diagnostic accuracy of MRI on predicting path logical response to NAC in breast cancer patients. The reported sensitivity and specificity of MRI were $63 \%$ and $91 \%$, respectively [18]. In our study, we found that when regression rate was higher than optimal critical value (81\%) after whole course of NAC, the sensitivity and specificity were predicted to be $90.4 \%$ and $89.7 \%$ which was close to the above research that only covered DCE-MRI. MRI included DWI and DCE-MRI was proved to have the ability to track small changes in tumor size and produce several indicators of treatment response in clinical studies over the last 10 years including semi-quantitative parameters, changes in lesion size, tumor regression rate, $\mathrm{ADC}$, choline concentration and water-to-fat ratio $[19,20]$. Our study revealed that the change rate of $\mathrm{ADC}_{\text {mean }}$ value had the highest diagnostic efficiency and proved that DWI and DCEMRI have prognostic value on NAC efficiency in breast cancer.
Taken as a whole, our study revealed that DWI and DCEMRI had diagnostic efficiency to NAC in patients with breast cancer and pretreatment $\mathrm{ADC}$ mean could be used to predict tumor regression rate. Our study had several limitations: firstly, we obtained sensitivity and specificity values based on a cutoff value determined by the population in this study. Use of this threshold in different populations would likely yield different sensitivity and specificity values. Secondly, all the patients recruited in this study were diagnosed with breast cancer and received treatment in our hospital from September 2011 to December 2014, which was not long enough for us to perform follow-up. The small sample size of the present study was also a limitation, which might likely lead to low power to detect differences between the effective and ineffective groups.

Acknowledgments: We would like to give our sincere appreciation to the reviewers for their helpful comments on this article.

\section{References}

[1] SENEVIRATNE S, CAMPBELL I, SCOTT N, KUPERHOMMEL M, KIM B et al. Adherence to adjuvant endocrine therapy: is it a factor for ethnic differences in breast cancer outcomes in New Zealand? Breast 2015; 24: 62-67. https:// doi.org/10.1016/j.breast.2014.11.011

[2] YOULDEN DR, CRAMB SM, DUNN NA, MULLER JM, PYKE CM et al. The descriptive epidemiology of female breast cancer: an international comparison of screening, incidence, survival and mortality. Cancer Epidemiol 2012; 36: 237-248. https://doi.org/10.1016/j.canep.2012.02.007 
[3] POOLE VL, MCCABE CJ. Iodide transport and breast cancer. J Endocrinol 2015; 227: R1-R12. https://doi.org/10.1530/JOE$\underline{15-0234}$

[4] HE Y, GONG J, WANG Y, QIN Z, JIANG Y et al. Potentially functional polymorphisms in aminoacyl-trna synthetases genes are associated with breast cancer risk in a Chinese population. Mol Carcinog 2015; 54: 577-583. https://doi. org/10.1002/mc.22128

[5] QUANG CT, LEBOUCHER S, PASSARO D, FUHRMANN L, NOURIEH $\mathrm{M}$ et al. The calcineurin/nfat pathway is activated in diagnostic breast cancer cases and is essential to survival and metastasis of mammary cancer cells. Cell Death Dis 2015; 6: e1658. https://doi.org/10.1038/cddis.2015.14

[6] MOUGALIAN SS, SOULOS PR, KILLELEA BK, LANNIN DR, ABU-KHALAF MM et al. Use of neoadjuvant chemotherapy for patients with stage $\mathrm{i}$ to iii breast cancer in the United States. Cancer 2015; 121: 2544-2552. https://doi. org/10.1002/cncr.29348

[7] CAUDLE AS, GONZALEZ-ANGULO AM, HUNT KK, LIU P, PUSZTAI L et al. Predictors of tumor progression during neoadjuvant chemotherapy in breast cancer. J Clin Oncol 2010; 28: 1821-1828. https://doi.org/10.1200/ LCO.2009.25.3286

[8] MICHISHITA S, KIM SJ, SHIMAZU K, SOTA Y, NAOI Y et al. Prediction of pathological complete response to neoadjuvant chemotherapy by magnetic resonance imaging in breast cancer patients. Breast 2015; 24: 159-165. https://doi. org/10.1016/j.breast.2015.01.001

[9] GOLD LS, LEE CI, DEVINE B, NELSON H, CHOU R et al. Imaging techniques for treatment evaluation for metastatic breast cancer. Effective Health Care Program. Technical Brief Number 17. Agency for Healthcare Research and Quality 2014. Contract No. 290-2012-00014-I.

[10] WILMES LJ, MCLAUGHLIN RL, NEWITT DC, SINGER L, SINHA SP et al. High-resolution diffusion-weighted imaging for monitoring breast cancer treatment response. Acad Radiol 2013; 20: 581-589. https://doi.org/10.1016/j.acra.2013.01.009

[11] OTO A, YANG C, KAYHAN A, TRETIAKOVA M, ANTIC T et al. Diffusion-weighted and dynamic contrast-enhanced MRI of prostate cancer: Correlation of quantitative MR parameters with Gleason score and tumor angiogenesis. AJR Am J Roentgenol 2011; 197: 1382-1390. https://doi.org/10.2214/AJR.11.6861

[12] FANGBERGET A, NILSEN LB, HOLE KH, HOLMEN MM, ENGEBRAATEN $\mathrm{O}$ et al. Neoadjuvant chemotherapy in breast cancer-response evaluation and prediction of response to treatment using dynamic contrast-enhanced and diffusionweighted MR imaging. Eur Radiol 2011; 21: 1188-1199. https://doi.org/10.1007/s00330-010-2020-3

[13] TALSMA K, VAN HAGEN P, GROTENHUIS BA, STEYERBERG EW, TILANUS HW et al. Comparison of the 6th and 7th editions of the UICC-AJCC TNM classification for esophageal cancer. Ann Surg Oncol 2012; 19: 2142-2148. https://doi.org/10.1245/s10434-012-2218-5

[14] RAZA S, GOLDKAMP AL, CHIKARMANE SA, BIRDWELL RL. Us of breast masses categorized as BI-RADS 3, 4, and 5: pictorial review of factors influencing clinical management. Radiographics 2010; 30: 1199-1213. https://doi.org/10.1148/ rg.305095144

[15] MCHUGH K, KAO S. Response evaluation criteria in solid tumours (RECIST): problems and need for modifications in paediatric oncology? Br J Radiol 2003; 76: 433-436. https:// doi.org/10.1259/bjr/15521966

[16] WU LM, HU JN, GU HY, HUA J, CHEN J et al. Can diffusionweighted $\mathrm{mr}$ imaging and contrast-enhanced $\mathrm{mr}$ imaging precisely evaluate and predict pathological response to neoadjuvant chemotherapy in patients with breast cancer? Breast Cancer Res Treat 2012; 135: 17-28. https://doi.org/10.1007/ s10549-012-2033-5

[17] PARK SH, MOON WK, CHO N, SONG IC, CHANG JM et al. Diffusion-weighted MR imaging: Pretreatment prediction of response to neoadjuvant chemotherapy in patients with breast cancer. Radiology 2010; 257: 56-63. https://doi.org/10.1148/ radiol.10092021

[18] YUAN Y, CHEN XS, LIU SY, SHEN KW. Accuracy of MRI in prediction of pathologic complete remission in breast cancer after preoperative therapy: a meta-analysis. AJR Am J Roentgenol 2010; 195: 260-268. https://doi.org/10.2214/ AJR.09.3908

[19] DANISHAD KK, SHARMA U, SAH RG, SEENU V, PARSHAD R et al. Assessment of therapeutic response of locally advanced breast cancer (LABC) patients undergoing neoadjuvant chemotherapy (NACT) monitored using sequential magnetic resonance spectroscopic imaging (MRSI). NMR Biomed 2010; 23: 233-241. https://doi.org/10.1002/ nbm.1436

[20] MCLAUGHLIN R, HYLTON N. MRI in breast cancer therapy monitoring. NMR Biomed 2011; 24: 712-720. https://doi. org/10.1002/nbm.1739 